\title{
Minimally-invasive cytoreduction in recurrent endometrial cancer: laparoscopic and uniportal video-assisted thoracoscopic surgery combined approach
}

Nicolò Bizzarri, ${ }^{1}$ Elisa Meacci, ${ }^{2}$ Felice Giuliante,${ }^{3,4}$ Valentina Ghirardi, ${ }^{1}$ Giovanni Scambia, ${ }^{1,4}$ Anna Fagotti ${ }^{1,4}$

${ }^{1}$ UOC Ginecologia Oncologica, Dipartimento per la salute della Donna e del Bambino e della Salute Pubblica, Fondazione Policlinico Universitario A. Gemelli, IRCCS, Roma, Italy ${ }^{2}$ Department of Thorarcic Surgery, Fondazione Policlinico Universitario A. Gemelli, IRCCS, Roma, Italy

${ }^{3}$ Department of Hepatobilary Surgery, Fondazione Policlinico Universitario A. Gemelli, IRCCS, Roma, Italy

${ }^{4}$ Università Cattolica del Sacro Cuore, Roma, Italy

\section{Correspondence to} Professor Giovanni Scambia, UOC Ginecologia Oncologica, Dipartimento per la salute della Donna e del Bambino e della Salute Pubblica, Fondazione Policlinico Universitario A. Gemelli, IRCCS, 00168 Roma, Italy; giovanni.scambia@ policlinicogemelli.it

Accepted 9 April 2020 Published Online First 23 April 2020

\section{Check for updates}

(C) IGCS and ESGO 2020. No commercial re-use. See rights and permissions. Published by BMJ.

To cite: Bizzarri N, Meacci E, Giuliante $\mathrm{F}$, et al. Int J Gynecol Cancer 2020;30:900.
Cytoreductive surgery for recurrent abdominal endometrial cancer is typically performed by laparotomy. ${ }^{12}$ Furthermore, thoracic procedures are usually performed with a thoracotomy approach. Recently, the uni-portal video-assisted thoracoscopic surgery (VATS) has been proposed as the new evolution of VATS, with even less morbidity and faster recovery. ${ }^{34}$ The video shows a patient with third abdominal and thoracic recurrence of endometrial cancer, managed with a combined laparoscopic and uni-portal VATS approach (Video 1). The patient is a 58-year-old woman, initially diagnosed with International Federation of Gynecology and Obstetrics (FIGO) stage IB grade 2 endometrioid endometrial adenocarcinoma. She had two previous recurrences both treated with minimally-invasive resection and chemotherapy. Eighteen months after the end of chemotherapy, a positron emission tomography (PET)computed tomography (CT) scan showed avid uptake in the liver capsule/right diaphragm and $1 \mathrm{~cm}$ right pulmonary nodule with mild uptake. She underwent laparoscopic full-thickness right diaphragm resection followed by uni-portal VATS with removal of right pleural nodules, diaphragm defect suturing, and an atypical resection of right middle lobe. Operation time was $180 \mathrm{~min}$ and estimated intra-operative blood loss was $80 \mathrm{mls}$. No macroscopic residual tumor was present at the end of the surgery. No intra-operative complication

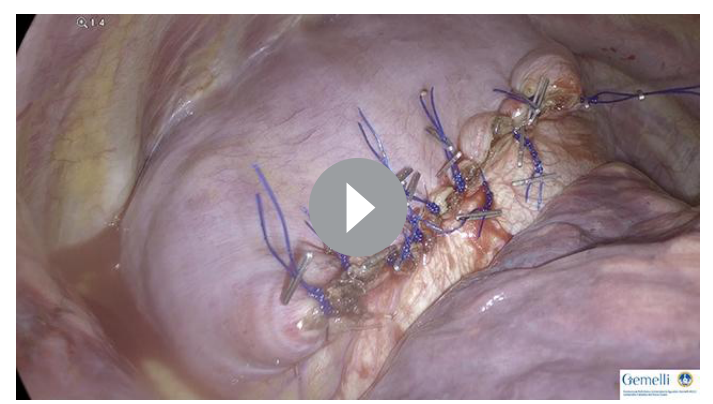

Video 1. Laparoscopic and uniportal videoassisted thoracoscopic surgery combined approach for recurrent endometrial cancer. was recorded. Post-operatively she developed a small right apical pneumothorax. She was discharged on day 4. Histology confirmed recurrent endometrial cancer in all specimens but in the pulmonary nodule (atypical hyperplasia). Adjuvant chemotherapy was started 14 days after the surgery. She is alive and disease-free 12 months after surgery. Minimally-invasive debulking abdominal and thoracic surgery to no residual disease for recurrent endometrial cancer, is feasible and ensures a rapid post-operative recovery to start medical treatment

Twitter Anna Fagotti @annafagottimd

Acknowledgements The Authors would like to acknowledge the surgical team of the Gynecologic Oncology Division at Policlinico Universitario Agostino Gemelli IRCCS, Rome, Italy.

Contributors NB: conceptualization, surgeon assistant, methodology, writing-original draft, video editing. EM: surgeon, review and editing manuscript. FG: surgeon, review and editing manuscript. VG: surgeon, review and editing manuscript, video editing. GS: conceptualization, surgeon, methodology, writing original draft. AF: conceptualization, methodology, writing original draft, video editing.

Funding The authors have not declared a specific grant for this research from any funding agency in the public, commercial, or not-for-profit sectors.

Competing interests None declared.

Patient consent for publication Not required.

Provenance and peer review Not commissioned; externally peer reviewed.

Data availability statement Data are available upon request

\section{REFERENCES}

1 Shikama A, Minaguchi T, Takao W, et al. Predictors of favorable survival after secondary cytoreductive surgery for recurrent endometrial cancer. Int J Clin Oncol 2019;24:1256-63.

2 Turan T, Tasci T, Karalok A, et al. Salvage cytoreductive surgery for recurrent endometrial cancer. Int J Gynecol Cancer 2015;25:1623-32.

3 Sihoe ADL. Uniportal video-assisted thoracic (VATS) lobectomy. Ann Cardiothorac Surg 2016;5:133-44.

4 Wang L, Liu D, Lu J, et al. The feasibility and advantage of uniportal video-assisted thoracoscopic surgery (VATS) in pulmonary lobectomy. BMC Cancer 2017;17:75. 